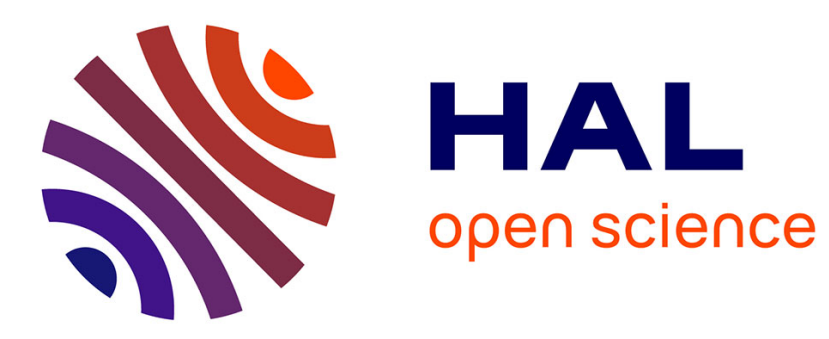

\title{
Évaluation des fuites thermiques par les fils de détecteurs dans les mesures de température en régime transitoire
}

J. Bransier, F. Collignon, H. Poncin

\section{To cite this version:}

J. Bransier, F. Collignon, H. Poncin. Évaluation des fuites thermiques par les fils de détecteurs dans les mesures de température en régime transitoire. Revue de Physique Appliquée, 1975, 10 (1), pp.17-26. 10.1051/rphysap:0197500100101700 . jpa-00243873

\section{HAL Id: jpa-00243873 https://hal.science/jpa-00243873}

Submitted on 1 Jan 1975

HAL is a multi-disciplinary open access archive for the deposit and dissemination of scientific research documents, whether they are published or not. The documents may come from teaching and research institutions in France or abroad, or from public or private research centers.
L'archive ouverte pluridisciplinaire HAL, est destinée au dépôt et à la diffusion de documents scientifiques de niveau recherche, publiés ou non, émanant des établissements d'enseignement et de recherche français ou étrangers, des laboratoires publics ou privés. 


\title{
ÉVALUATION DES FUITES THERMIQUES PAR LES FILS DE DÉTECTEURS DANS LES MESURES DE TEMPÉRATURE EN RÉGIME TRANSITOIRE
}

\author{
J. BRANSIER, F. COLLIGNON et H. PONCIN
}

Laboratoire de Recherches sur les Echanges Thermiques, Université de Paris VI, 4, place Jussieu 75230 Paris Cedex 05, France

\author{
(Reçu le 11 juillet 1974)
}

\begin{abstract}
Résumé. - Les fils qui assurent la liaison électrique entre les capteurs de température et la chaîne de mesure constituent des puits thermiques dont l'existence modifie les phénomènes à étudier. Trois ensembles concrets sont analysés en vue de déterminer l'ordre de grandeur de la perturbation apportée par le dispositif métrologique et d'en déduire les dispositions à prendre pour optimiser ce dispositif. Cette analyse montre que - d'une façon générale - les résultats obtenus en régime établi sont très différents de ceux que l'on obtient au cours d'une évolution provoquée par la rupture brusque d'un équilibre thermique. Dans le cas de phénomènes évolutifs, il arrive que - en partant d'hypothèses très voisines les unes des autres - on aboutisse à des conclusions très différentes en ce qui concerne le choix des fils de liaison et la détermination de la partie utilisable des enregistrements. Il n'existe donc pas de recette universelle mais chaque problème concret peut être résolu suivant une technique générale comportant l'élaboration d'un modèle, le traitement de ce modèle par le calcul opérationnel, un calcul numérique par ordinateur et la construction d'abaques, cotées en fonction des coefficients d'échange.

Dans certains cas, on peut - en utilisant des fonctions majorantes - obtenir directement la réponse aux questions posées, sans avoir à utiliser le calcul par ordinateur.

Abstract. - The electrical wires connecting the temperature sensors and the measuring system form a thermal sink whose existence influences the investigated phenomena. Three concrete examples are analyzed with the aim of estimating the disturbance due to the metrological device so as to deduce the arrangements necessary to optimize that device. Such an analysis reveals that as a rule, the results in a steady heat flow are very different from the results obtained in an evolution provoked by the sudden break of the calorific balance. In the case of non-steady state phenomena, very similar assumptions may well lead to widely different conclusions as regards the choice of the connecting wires and the analysis of the recordings. There is therefore no universal principle, but each concrete problem can be solved through a general method involving the setting up of a pattern, its treatment through operational calculus, a digital processing, and the drawing of charts dimensioned in terms of transfer factors. In some cases, a direct answer to the questions asked can be obtained through the use of majoring functions, without resorting to data processing.
\end{abstract}

1. Parmi toutes les difficultés que l'on rencontre lorsque l'on veut effectuer des mesures de température, figurent celles qui sont provoquées par l'existence de fils de jonction entre les capteurs et les instruments de mesure proprement dits car les fuites calorifiques qu'ils provoquent modifient le phénomène à étudier.

Cet effet perturbateur - que l'on qualifie d'effet de puits - est bien signalé dans les traités de métrologie et dans certains aide-mémoire $[1,2]$ mais, comme il ne peut pas être chiffré de manière systématique, il subsiste à son sujet une incertitude qu'il convient de lever dans chaque cas particulier.

Il est cependant indispensable d'en avoir tenu compte pour pouvoir certifier que l'on a obtenu une précision donnée. D'autre part, cette évaluation permet d'opti- miser les dispositifs expérimentaux et les techniques d'interprétation des enregistrements.

C'est pourquoi nous avons pensé qu'il était intéressant de communiquer les constatations que nous avons faites à ce sujet au cours d'études récentes. En effet, la méthode que nous avons adoptée, peut être étendue, dans son principe, à des montages très différents les uns des autres et constituer de ce fait un guide ayant une certaine portée.

2. Etude sur l'effusivité. - Pour en exposer le principe nous avons retenu trois appareillages dont l'optimisation nous a conduits - comme nous le verrons à adopter finalement des dispositions différentes.

Le premier problème que nous considérerons concerne un appareil et une technique expérimentale 
mis au point par l'un de nous, au cours d'études sur l'effusivité des isolants thermiques.

Le dispositif est décrit et analysé dans les ouvrages cités en référence $[3,4]$ dans un esprit très différent de celui que nous envisageons actuellement. Pour illustrer les considérations générales qui font l'objet de cette note, il nous suffit - ici - de signaler que l'appareil se compose essentiellement (cf. Fig. 1) de deux enceintes chauffantes régulées et de deux éléments cylindriques dont nous préciserons ultérieurement les dimensions (diamètre $D$ et épaisseur $e$ ). L'un d'entre eux est fabriqué à partir d'une substance à forte conductivité thermique (cuivre par exemple) l'autre à partir d'une substance à faible conductivité (plexiglas par exemple). Dans la suite de cet exposé - et conformément d'ailleurs aux rôles qu'ils jouent respectivement dans l'étude en question - le premier élément sera désigné sous le nom de capteur et symbolisé par l'initiale « $\mathrm{C}$ ». Le second sera désigné sous le nom «d'échantillon» et symbolisé par l'initiale « $\mathrm{E}$ ».

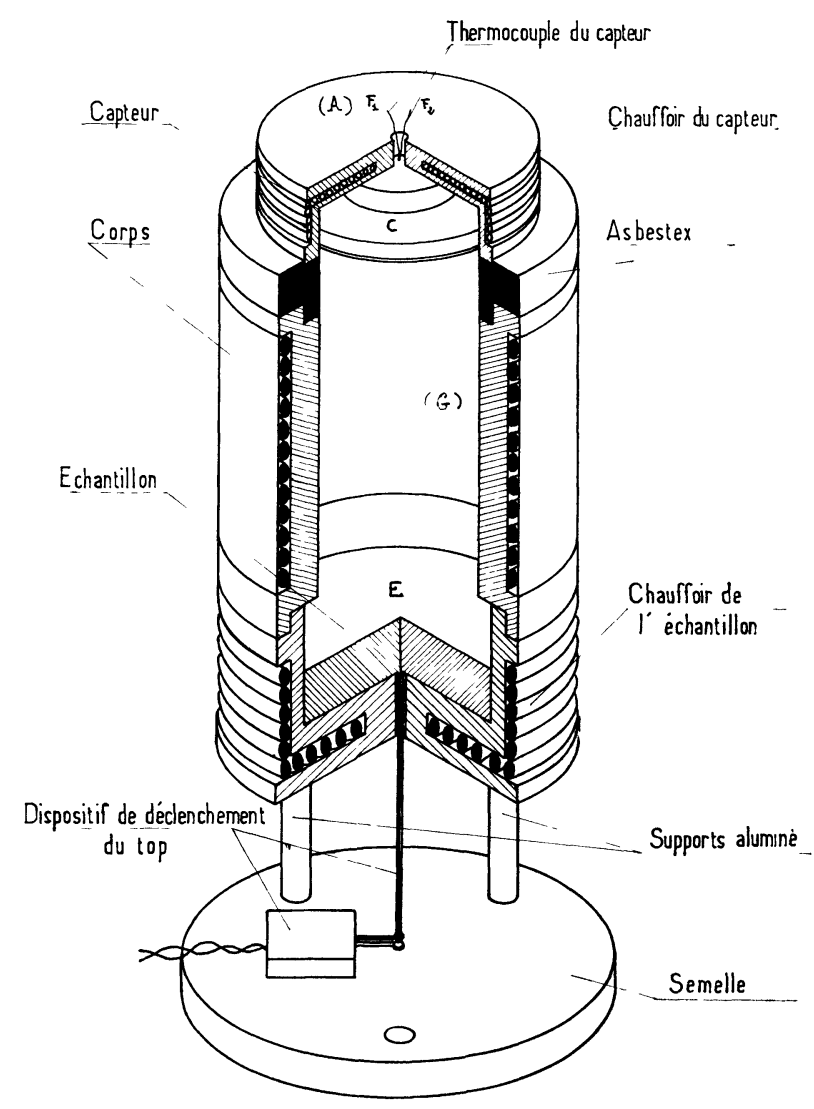

Fig. 1. - Schéma d'un effusimètre.

Un système de garde (Fig. 2) permet de supprimer tout échange de chaleur entre le capteur " $\mathrm{C}$ » et le milieu extérieur, sauf à travers la section droite inférieure du capteur et à travers la jonction entre ce capteur et les fils du thermocouple qu'il faut bien incorporer au capteur pour enregistrer les thermogrammes. Toutes les dispositions nécessaires ont été prises pour assurer un contact thermique pratiquement parfait entre la jonction de mesure du thermocouple et le capteur.
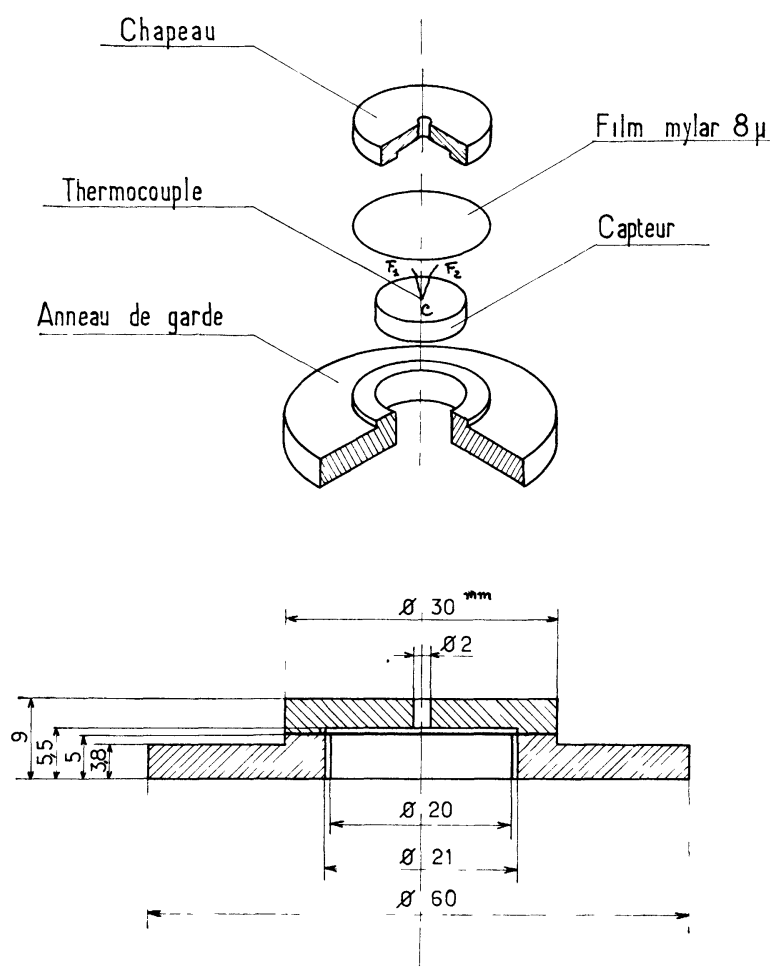

FIG. 2. - Schéma de principe de l'ensemble du capteur et des dispositifs de garde.

Pour désigner chacun des fils du couple ou le fil équivalent que nous définirons ultérieurement, nous utiliserons les symboles $« F_{1} », 《 F_{2} »$ et $« F$ $F$. Toutes les grandeurs considérées seront donc - suivant les cas - affectées de l'un des indices $« \mathrm{C} »,\langle\mathrm{E} »$ ou « $\mathrm{F} »\left({ }^{1}\right)$.

La technique opératoire est la suivante : dans une phase préparatoire le capteur est maintenu à la partie supérieure du dispositif. Les échanges thermiques intéressent alors le capteur $" \mathrm{C} »$, le chauffoir supérieur, le gaz intérieur à l'enceinte et les fils $" F$ ». Ceux-ci sont reliés aux instruments de mesure en traversant un milieu dont nous représenterons l'influence thermique à l'aide d'une température ambiante de référence $T(\mathrm{~A})$ (de l'ordre de $20^{\circ} \mathrm{C}$ ). On la détermine en tenant compte des effets convectifs et des effets radiatifs. Le dispositif de régulation thermique permet d'obtenir - au bout d'un certain temps - un état d'équilibre dans lequel l'ensemble du capteur atteint une température que nous désignerons par $T(C \mid .0)\left(40{ }^{\circ} \mathrm{C}\right.$ par exemple). Simultanément l'élément « $\mathrm{E}$ » atteint du fait des échanges avec le chauffoir inférieur et avec l'air contenu dans le corps de l'appareil une température d'équilibre un peu infé-

(1) On trouvera à la fin de cet article un tableau d'ensemble concernant les symboles utilisés. Tous les nombres donnés sont exprimés en unités légales, sauf indication contraire. 
rieure à $T(\mathrm{C} \mid .0)\left(30^{\circ} \mathrm{C}\right.$ par exemple) que nous désignerons par $T(\mathrm{E} \mid .0)$. Lorsque les deux états d'équilibre sont obtenus, on déclenche un dispositif qui permet de réaliser de façon quasi instantanée un contact matériel entue le capteur " $C$ » et l'échantillon « $E$ ».

Dans la deuxième phase de l'expérience, les échanges thermiques à considérer concernent :

1) la liaison thermique $(C \leftrightarrow E)$ qui s'effectue sur la section droite inférieure du capteur et sur la section droite supérieure de l'échantillon,

2) la liaison $(C \leftrightarrow F)$ qui s'effectue sur la section de raccordement du thermocouple au capteur,

3) la liaison $(\mathrm{F} \leftrightarrow \mathrm{G})$ qui s'effectue à travers la surface latérale entre les fils « $F$ » et le gaz « $G$ » contenu dans l'enceinte. Cette liaison peut être caractérisée par une température ambiante de référence $T(\mathrm{G})$ différente de la température $T(\mathrm{~A})$ que nous avions à considérer dans la phase préliminaire. Pour fixer les idées, nous lui affecterons la valeur numérique $T(\mathrm{G})=35^{\circ} \mathrm{C}$.

La détermination de l'effusivité de l'échantillon s'effectue à partir de l'étude des thermogrammes relatifs au capteur " $\mathrm{C}$ ». Etant donné la valeur de la conductivité du cuivre et les dimensions du capteur il est légitime d'admettre que la répartition des températures à l'intérieur du capteur est uniforme quel que soit l'instant « $t$ " considéré. Dans ces conditions, nous n'avons à considérer qu'un thermogramme chronologique que nous représenterons par $T(\mathrm{C} \mid \cdot t)$.

Pour que l'effet d'effusivité ne soit pas masqué par l'effet de diffusivité, on ne doit prendre en considération que les parties du thermogramme qui correspondent à une période très courte suivant immédiatement la rupture de l'équilibre thermique. Dans les expériences faites, le refroidissement du capteur pendant cette période ne dépassait pas quelques dixièmes de degré. On conçoit que, dans de telles conditions, il faut analyser avec soin tous les phénomènes qui peuvent avoir une influence sur l'évolution thermique du capteur.

Il nous faut en particulier répondre aux questions suivantes :

1) en quoi les thermogrammes enregistrés diffèrentils des thermogrammes (impossibles à obtenir expérimentalement) que l'on obtiendrait si la liaison $(C \leftrightarrow E)$ constituait la seule liaison thermique entre le capteur et le milieu extérieur?

2) quel type de thermocouples faut-il adopter (nature des thermoéléments, nature des gaines, diamètre des fils et de la gaine) pour obtenir une bonne précision dans la détermination du coefficient d'effusivité en remplissant au mieux les autres conditions imposées (par exemple des conditions de tenue mécanique) ?

3) quelle portion des thermogrammes enregistrés devrons-nous prendre en considération pour le dépouillement?
Pour répondre à ces questions, il convient de définir des modèles thermocinétiques susceptibles de représenter de façon satisfaisante chacun des éléments du système. Comme nous l'avons déjà dit le nombre de Biot attaché au capteur " $C$ » dans les conditions expérimentales définies ci-dessus, est suffisamment faible pour que - conformément aux résultats indiqués dans tous les traités de thermocinétique - on puisse considérer une répartition des températures uniforme dans toute son étendue.

La valeur de la température sur la surface de contact (C-E) est donc égale à celle qui correspond - au même instant - au contact (C-F) c'est-à-dire à la section $x=0$ des fils « $\mathrm{F}$ ».

D'autre part - à condition de n'utiliser qu'une partie des enregistrements - il est légitime de représenter l'échantillon « $E$ » en utilisant le modèle thermocinétique du mur semi-infini. Les thermogrammes correspondant ne font intervenir qu'une variable d'espace, à savoir la distance $y$ du point considéré à la face supérieure de l'échantillon. Nous les représenterons par $T(\mathrm{E} \mid y, t)$. La liaison thermique (C-E) fait intervenir une résistance de contact qui a été prise en considération dans l'étude dont nous avons parlé mais en l'introduisant ici, nous ne ferions que compliquer les calculs et l'écriture des formules sans apporter d'informations supplémentaires sur la méthode générale. C'est pourquoi nous supposerons a priori qu'il s'agit d'une liaison parfaite au point de vue thermique. Cette hypothèse correspond d'ailleurs à certaines expériences que nous avons faites en intercalant entre « $\mathrm{C}$ » et « $\mathrm{E}$ » un fluide convenablement choisi.

Il est plus difficile d'obtenir - pour les fils - un modèle thermocinétique qui calque la réalité physique tout en permettant d'effectuer des calculs. Lorsque les fils $" F_{1}$ » et $" F_{2}$ " sont séparés il est légitime d'adopter pour chacun d'eux le modèle que les thermocinéticiens désignent sous le nom de modèle de la barre semi-infinie. Les échanges entre $《 F$ » et $\| \mathrm{G}$ » s'effectuent en effet de façon uniforme sur tout le périmètre du fil et on peut les caractériser par une relation linéaire entre le flux et la température du fil dans la section d'abscisse $x$ en prenant en considération la température ambiante de référence

$$
[T(\mathrm{~A}) \text { ou } T(\mathrm{G})] \text {, }
$$

les caractéristiques physiques du thermoélément (conductivité $\lambda$, capacité calorifique volumique $\gamma=\rho c$, diffusivité $a$, ou effusivité $b$ ), la géométrie (diamètre $d$ ) et la nature de la gaine. Si l'on désigne par $h$ le coefficient d'échange, les paramètres représentatifs seront

- soit le nombre de Biot

$$
B_{i}=\frac{h d(\mathrm{~F})}{\lambda(\mathrm{F})}
$$

(sans dimension), 
- soit le nombre

$$
m^{2}=\frac{4 h}{\rho c(\mathrm{~F}) \cdot d(\mathrm{~F})}=4 B i \frac{a(F)}{d^{2}(\mathrm{~F})}
$$

qui est homogène à $1 / t$.

On peut être plus embarrassé lorsqu'il s'agit de représenter un couple thermoélectrique du type thermocoax. Dans ce cas la section présente en effet l'aspect représenté sur la figure 3. Comme leurs

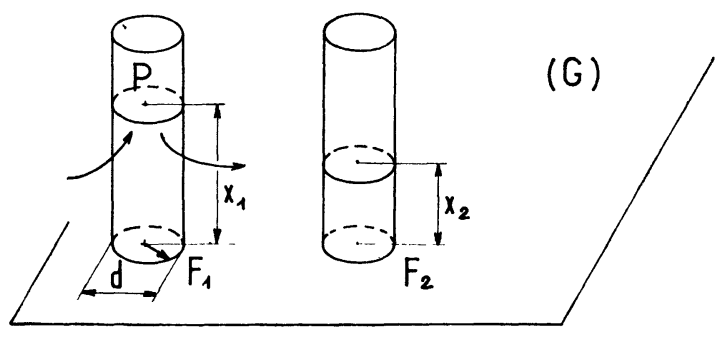

$$
d_{F}=d \sqrt{2}
$$
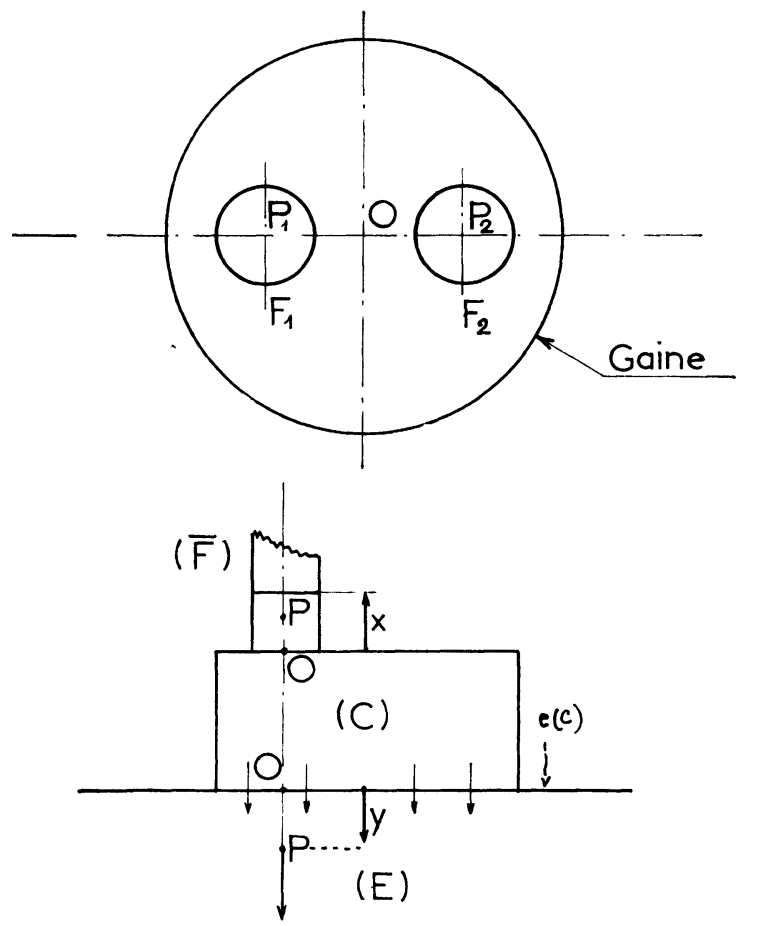

Fig. 3. - Définition du fil équivalent.

substances n'ont pas les mêmes caractéristiques thermophysiques, les fils « $F_{1}$ » et $" F_{2}$ » ne conduisent pas la chaleur de la même façon et il en résulte qu'à une certaine distance de la jonction les températures aux points « $P_{1}$ » et « $P_{2}$ » ne sont pas rigoureusement les mêmes. D'autre part, l'ensemble n'admet pas la symétrie de révolution de centre $O$ et il semble difficile en conséquence de définir la température moyenne dans la section et la température superficielle de la gaine qui doit être prise en considération pour calculer les échanges thermiques entre les fils et le milieu ambiant, mais ce sont là des circonstances qui se rencontrent fréquemment en thermique où l'on a souvent affaire à des milieux hétérogènes et l'expérience a montré que l'on pouvait se tirer d'affaire en travaillant sur les modèles classiques à condition d'affecter ultérieurement des valeurs numériques convenables aux paramètres qu'ils introduisent. Nous avons vérifié qu'il en était bien ainsi dans le problème que nous étudions actuellement. En résumé, nous pouvons représenter l'ensemble du thermocouple par un fil unique que nous appellerons fil équivalent (symbole «F $)$ ). Toutefois comme le coefficient $h$ qui caractérise sa liaison avec le milieu environnant (A) ne peut pas être chiffré $a$ priori avec précision, il faut envisager pour $h$ un certain nombre de valeurs numériques situées à l'intérieur d'une certaine fourchette.

Pour fixer les idées au point de vue des ordres de grandeurs, nous considérerons un couple thermoélectrique chromel-alumel. Les valeurs caractéristiques sont alors $\rho\left(\mathrm{F}_{1}\right)=8730, \mathrm{C}\left(\mathrm{F}_{1}\right)=447, \lambda\left(\mathrm{F}_{1}\right)=19$, $\rho\left(\mathrm{F}_{2}\right)=8600, \mathrm{C}\left(\mathrm{F}_{2}\right)=523, \lambda\left(\mathrm{F}_{2}\right)=30$. En première approximation, on adoptera pour le fil équivalent dont le diamètre $d(\mathrm{~F})$ est relié au diamètre nominal par $d(\mathrm{~F})=d \sqrt{2}$, les valeurs moyennes de $(\rho c)$ et de $\lambda$.

Ayant ainsi défini les hypothèses de travail il est facile - en adoptant des méthodes classiques en thermocinétique - de déterminer analytiquement les différents thermogrammes.

$\mathrm{Au}$ cours de la phase préliminaire de mise en température pendant laquelle le capteur et l'échantillon atteignent respectivement un état d'équilibre thermique aux niveaux $T(\mathrm{C} \mid .0)$ et $T(\mathrm{E} \mid .0)$ le fil « $\mathrm{F}$ » équivalent au thermocouple réel atteint un état stationnaire défini par:

$$
\begin{aligned}
T(\mathrm{~F} \mid .0)=T(\mathrm{~A})+[T(\mathrm{C} \mid .0)-T(\mathrm{~A})] \times \\
\times \exp \left[-\frac{m x}{\sqrt{a(\mathrm{~F})}}\right] .
\end{aligned}
$$

Au cours de l'expérience proprement dite, le capteur se refroidit sous l'effet combiné des échanges avec l'échantillon « $E$ » d'une part et avec les fils « $F$ » d'autre part.

Si nous désignons par $\gamma(c)$ la capacité calorifique volumique du capteur et par $\varphi(\mathrm{E} \rightarrow \mathrm{C})$ et $\varphi(\mathrm{F} \rightarrow \mathrm{C})$ les densités superficielles de flux qui définissent les échanges en question, nous pouvons exprimer le bilan thermique sous la forme

$$
\begin{aligned}
& \pi \gamma(\mathrm{C}) \cdot e(\mathrm{C}) D^{2}(\mathrm{C}) \frac{\mathrm{d} T(\mathrm{C})}{\mathrm{d} t}= \\
& \quad=\pi D^{2}(\mathrm{C}) \varphi(\mathrm{E} \rightarrow \mathrm{C})+2^{\prime} \pi D^{2}(\mathrm{~F}) \varphi(\mathrm{F} \rightarrow \mathrm{C}) .
\end{aligned}
$$

Nous obtiendrons les deux autres relations qui se révèlent nécessaires pour la détermination analytique du thermogramme $T(\mathrm{C} \mid . t)$ en considérant les évolutions thermiques des éléments $« E »$ et $" F$ ». Le 
modèle adopté pour la représentation de l'échantillon permet d'écrire les relations suivantes :

$$
\begin{gathered}
\frac{\partial}{\partial t}[T(\mathrm{E} \mid y, t)]=a(\mathrm{E}) \cdot \frac{\partial^{2}}{\partial y^{2}} \cdot[T(\mathrm{E} \mid y, t)] \\
\varphi(\mathrm{E} \rightarrow \mathrm{C})=\lambda(\mathrm{E}) \cdot \frac{\partial}{\partial y}[T(\mathrm{E} \mid y, t)]_{y=0} \\
T(\mathrm{E} \mid \infty, t)=T(\mathrm{E} \mid \cdot 0) \\
T(\mathrm{E} \mid 0, t)=T(\mathrm{C} \mid \cdot t) .
\end{gathered}
$$

Le procédé le plus commode pour effectuer les calculs consiste à considérer la correspondance qui existe entre les thermogrammes $T(\mathrm{E} \mid y, t)$ et leurs images de Laplace (que nous représenterons par $\mathcal{L}[T(\mathrm{E} \mid y, t)]$. En transformant l'éq. (3) on obtient :

$$
a(\mathrm{E}) \frac{\partial^{2}}{\partial y^{2}}\{\mathcal{L}[T(\mathrm{E})]\}-p \mathcal{L}[T(\mathrm{E})]=-T(\mathrm{E} \mid .0)
$$

d'où l'on déduit l'expression

$$
\begin{array}{r}
\mathcal{L}[T(E)]=T(E \mid .0) \cdot \frac{1}{p}+F(p) \exp \left[-\frac{q}{\sqrt{a(E)}} y\right] \\
\text { où } q=\sqrt{p}
\end{array}
$$

et dans laquelle $F(p)$ représente une fonction de l'argument « $p$ », qui n'est pas déterminée par l'équation de diffusion mais par les conditions aux limites. On a en effet, d'après (4) :

$$
\mathcal{L}[\varphi(\mathrm{E} \rightarrow \mathrm{C})]=-b(\mathrm{E}) \cdot q F(p)
$$

et d'après (6) et (9) :

$$
F(p)=\mathcal{L} \cdot[T(\mathrm{C} \mid t)]-T(\mathrm{E} \mid \cdot 0) \frac{1}{p}
$$

$\mathcal{L}[\varphi(E \rightarrow \mathrm{C})]=b(E) \cdot\left\{\frac{1}{q} T(\mathrm{E} \mid \cdot 0)-q \mathcal{L}[T(\mathrm{C} \mid \cdot t)]\right\}$

L'étude de l'évolution de l'élément « $F$ » s'effectue de la même façon en utilisant les équations thermocinétiques correspondant au modèle de la barre semiinfinie. On exprime ainsi le bilan énergétique sous la forme :

$$
\begin{aligned}
\frac{\partial}{\partial t} \cdot\{T(\mathrm{~F} \mid x, t)\}= & a(\mathrm{~F}) \frac{\partial^{2}}{\partial x^{2}} \cdot[T(F \mid x, t)]+ \\
& +m^{2}[T(G)-T(F \mid x, t)]
\end{aligned}
$$

la condition initiale sous la forme :

$$
\begin{array}{r}
T(F \mid x, 0)=T(\mathrm{~A})+[T(\mathrm{C} \mid .0)-T(\mathrm{~A})] \times \\
\times \exp \left[-\frac{m x}{\sqrt{a(F)}}\right]
\end{array}
$$

et enfin la liaison thermique $(\mathrm{C} \leftrightarrow \mathrm{F})$ sous la forme :

$$
\lim _{x \rightarrow 0}[T(\mathrm{~F} \mid x, t)]=T(\mathrm{C} \mid \cdot t) \text {. }
$$

Si l'on cherche une solution qui reste finie lorsque $x$ augmente indéfiniment, on obtient l'expression :

$$
\begin{gathered}
\mathcal{L}[T(\mathrm{~F} \mid x, t)]=G(p) \exp \left[-\frac{\sqrt{p+m^{2}}}{\sqrt{a(\mathrm{~F})}} \cdot x\right]+ \\
+\frac{T(\mathrm{~A})}{p+m^{2}}+\frac{T(\mathrm{C} \mid \cdot 0)-T(\mathrm{~A})}{p} \\
\quad \times \exp \left[-\frac{m x}{\sqrt{a(\mathrm{~F})}}\right]+\frac{m^{2}}{p+m^{2}} \frac{T(G)}{p}
\end{gathered}
$$

qui correspond à :

$$
T[(\mathrm{~F} \mid \infty, t)]=T(\mathrm{G})+[T(\mathrm{~A})-T(\mathrm{G})] \exp \cdot\left(-m^{2} t\right) .
$$

En exprimant que la liaison $(\mathrm{C} \leftrightarrow \mathrm{F})$ est une liaison parfaite au point de vue thermique, on obtient :

$$
\begin{aligned}
& G(p)=\mathcal{L}[T(\mathrm{C} \mid . t)]- \\
& -T(\mathrm{C} \mid .0) \frac{1}{p}-m^{2}[T(\mathrm{G})-T(\mathrm{~A})] \frac{1}{p\left(p+m^{2}\right)} .
\end{aligned}
$$

Dans ces conditions, le flux échangé avec le capteur est défini par :

$$
\begin{aligned}
& \mathcal{L}[\varphi(\mathrm{C} \rightarrow \mathrm{F})]= \\
= & b(\mathrm{~F})\left\{\sqrt{p+m^{2}} G(p)+\frac{m}{p}[T(\mathrm{C} \mid .0)-T(\mathrm{~A})]\right\} .
\end{aligned}
$$

Ayant ainsi relié les flux à la fonction $\mathcal{L}[T(\mathrm{C} \mid . t)]$ et aux données fondamentales, nous pouvons exploiter le bilan énergétique du capteur.

Après transformation l'équation (2) s'écrit :

$$
\begin{aligned}
\gamma(\mathrm{C}) . e(\mathrm{C}) & \{p \mathcal{L}[T(\mathrm{C} \mid . t)]-T(\mathrm{C} \mid .0)\}= \\
& =\mathcal{L} \varphi(\mathrm{E} \rightarrow c)+2\left[\frac{d(\mathrm{~F})}{d(\mathrm{C})}\right]^{2} \mathcal{L} \varphi(\mathrm{F} \rightarrow \mathrm{C})
\end{aligned}
$$

et, en tenant compte de (10) et de (11) on obtient finalement l'expression :

$$
\begin{gathered}
\mathcal{L}\left[\frac{T(\mathrm{C} \mid \cdot t)-T(\mathrm{C} \mid \cdot 0)}{T(\mathrm{C} \mid \cdot 0)}\right]\left[p+\alpha q+\varepsilon \sqrt{p+m^{2}}\right]= \\
=\frac{\alpha}{q} \cdot \frac{T(\mathrm{E} \mid \cdot 0)-T(\mathrm{C} \mid .0)}{T(\mathrm{C} \mid \cdot 0)}-\varepsilon \frac{m}{p} \frac{T(\mathrm{C} \mid .0)-T(\mathrm{~A})}{T(\mathrm{C} \mid .0)}+ \\
+\frac{\varepsilon m^{2}}{p \sqrt{p+m^{2}}} \cdot \frac{T(\mathrm{G})-T(\mathrm{~A})}{T(\mathrm{C} \mid \cdot 0)}
\end{gathered}
$$

qui contient trois paramètres $\alpha, \varepsilon$ et $m$ s'exprimant en fonction des grandeurs physiques considérées précédemment par les relations

$$
\begin{gathered}
\alpha=\frac{b(\mathrm{E})}{\gamma(\mathrm{C}) \cdot e(\mathrm{C})} \quad \varepsilon=2 \frac{b(\mathrm{~F})}{\gamma(\mathrm{C}) \cdot e(\mathrm{C})}\left[\frac{d(\mathrm{~F})}{d(\mathrm{C})}\right]^{2} \\
m^{2}=\frac{4 h}{\gamma(\mathrm{F}) d(\mathrm{~F})} .
\end{gathered}
$$

Ils sont tous homogènes à $1 / \sqrt{t}$. 
Dans l'exemple choisi le paramètre $\alpha$ qui caractérise l'effet de contact est de l'ordre de $2 \times 10^{-2}$ le paramètre $\varepsilon$ qui carac térise l'effet de puits est de l'ordre de $2 \times 10^{-5}$ et le paramètre $m$, qui caractérise les échanges latéraux de l'ordre de $\sqrt{h} \cdot 10^{-1}$.

Il en résulte que l'on peut mettre en évidence l'importance relative de ces différents effets en utilisant un développement de $\mathcal{L}$ limité au terme en $\varepsilon$. Pour interpréter les résultats il sera commode d'utiliser la forme adimensionnelle

$$
\begin{aligned}
& \mathcal{L}\left[\frac{T(\mathrm{C} \mid . t)-T(\mathrm{C} \mid .0)}{T(\mathrm{C} \mid .0)}\right]= \\
&=\alpha\left[\frac{1}{p(q+\alpha)}-\varepsilon \frac{\sqrt{p+m^{2}}}{p q(q+\alpha)^{2}}\right] \frac{T(E \mid .0)-T(\mathrm{C} \mid .0)}{T(\mathrm{C} \mid .0)}- \\
&-\frac{\varepsilon m}{p q(q+\alpha)} \frac{T(\mathrm{C} \mid .0)-T(\mathrm{~A})}{T(\mathrm{C} \mid .0)}+\frac{\varepsilon m^{2}}{p q(q+\alpha) \sqrt{p+m^{2}}} \\
& \quad \times \frac{T(\mathrm{G})-T(\mathrm{~A})}{T(\mathrm{C} \mid .0)}=\mathfrak{L}^{*}(\alpha, \varepsilon, m) .
\end{aligned}
$$

Il est possible de calculer les originaux de chacun des termes qui figurent dans cette formule soit directement soit en utilisant le produit de convolution. On peut donc, à partir de données numériques précises, mettre en évidence la différence qui existe entre le thermogramme obtenu expérimentalement et le modèle qui correspondrait à $\varepsilon=0$, c'est-à-dire au cas où les fils de liaison transmettraient le signal sans le perturber, mais comme ce calcul est long, on est conduit à limiter son emploi aux seuls cas où il est indispensable de le faire. Or, on peut parfois l'éviter en remarquant que l'on surestime l'effet de puits si l'on néglige l'effet de contact c'est-à-dire si l'on néglige $\alpha$ dans la formule (19).

En effet - dans les conditions de l'expérience - le contact $(C \leftrightarrow E)$ provoque un refroidissement du capteur, qui diminue l'écart de température existant entre le capteur et les fils de liaison. Il en résulte qu'il est inutile d'effectuer le calcul complet si l'on constate $a$ priori que l'ordre de grandeur de la différence qui existe entre le thermogramme $(0, \varepsilon, m)$ et le thermogramme $(\alpha, 0, m)$ correspond déjà au degré de précision recherché pour la mesure.

Ceci nous conduit à considérer le thermogramme adimensionnel dont l'image est définie par

$$
\begin{aligned}
\mathcal{L}^{*}(0, \varepsilon, m)=- & \frac{\varepsilon m}{p^{2}} \frac{T(\mathrm{C} \mid .0)-T(\mathrm{~A})}{T(\mathrm{C} \mid .0)}+ \\
& +\frac{\varepsilon m^{2}}{p^{2} \sqrt{p+m^{2}}} \frac{T(\mathrm{G})-T(\mathrm{~A})}{T(\mathrm{C} \mid .0)}
\end{aligned}
$$

et dont il est facile de déterminer l'original. On sait en effet que l'original de $1 / p^{2}$ est $t$. Pour calculer l'original du deuxième terme on peut effectuer dans le plan de la variable $p$ la translation $p+m^{2}=p^{\prime}$.
Après un développement en éléments simples, on obtient dans cet espace l'image :

$$
\begin{aligned}
\frac{m^{2}}{q^{\prime}\left[q^{\prime 2}-m^{2}\right]^{2}}= & \\
=\frac{1}{m^{2}} \cdot \frac{1}{q^{\prime}} & +\frac{1}{4 m}\left[\frac{1}{\left(q^{\prime}-m\right)^{2}}-\frac{1}{\left(q^{\prime}+m\right)^{2}}\right]- \\
& -\frac{1}{2 m^{2}}\left[\frac{1}{q^{\prime}-m}+\frac{1}{q^{\prime}+m}\right] .
\end{aligned}
$$

On connaît les originaux qui correspondent à chacun des termes de cette formule si bien que l'on peut expliciter $\mathcal{L}^{*}(0, \varepsilon, m)$ sous la forme :

$$
\begin{aligned}
& \delta T^{*}(0, \varepsilon, m)= \\
&=-\varepsilon m \frac{T(\mathrm{C} \mid .0)-T(\mathrm{~A})}{T(\mathrm{C} \mid .0)} t+\varepsilon \frac{T(\mathrm{G})-T(\mathrm{~A})}{T(\mathrm{C} \mid .0)} \times \\
& \times\left[\frac{\sqrt{t}}{\sqrt{\pi}} \exp \left(-m^{2} t\right)+\frac{1-2 m^{2} t}{2 m} \Theta(m \sqrt{t})\right]
\end{aligned}
$$

dans laquelle $\Theta$ représente, la fonction

$$
\Theta(x)=\frac{2}{\sqrt{\pi}} \int_{0}^{x} \exp \left(-u^{2}\right) \mathrm{d} u
$$

dont on possède d'excellentes tables numériques.

On a d'autre part :

$$
\begin{aligned}
& \delta T^{*}(\alpha, 0, m)=\frac{T(\mathrm{E} \mid .0)-T(\mathrm{C} \mid .0)}{T(\mathrm{C} \mid .0)} \times \\
& \quad \times\left[1-\exp \left(-\alpha^{2} t\right)+\exp \left(-\alpha^{2} t\right) \Theta(\alpha \sqrt{t})\right]
\end{aligned}
$$

Il est facile de comparer les expressions (24) et (25) même si l'on ne dispose d'aucun moyen matériel de calcul. C'est ainsi que dans une expérience sur le plexiglas dans laquelle on utilise un capteur en cuivre équipé d'un couple chromel-alumel constitués par des fils de $1 / 10 \mathrm{~mm}$ isolés sous soie et des gaines de soie paraffinées, on obtient respectivement à l'instant $t=1 \mathrm{~s}$ les valeurs $5 \times 10^{-6}$ et $7 \times 10^{-3}$. On voit que, dans ces conditions, on peut affirmer $a$ priori qu'il n'y a pas lieu de prendre en considération l'effet de puits provoqué par le thermocouple.

Pour mettre en évidence dans tous les cas, l'influence de l'isolation des fils nous avons calculé l'expression

$$
\delta T_{*}=\left[\frac{T(\mathrm{C} \mid .0)-T(\mathrm{C} \mid \cdot t)}{T(\mathrm{C} \mid .0)}\right]_{\alpha=0}
$$

pour différentes valeurs du coefficient d'échange $h(\mathrm{~F} \rightarrow \mathrm{G})$ en utilisant - cette fois - l'expression exacte qui correspond à la formule (19). Les programmes FORTRAN établis à cet effet ont été traités par l'ordinateur CII 10070 de l'atelier d'informatique des Universités de Paris-Centre. Les résultats obtenus sont représentés sur les figures 4 et 5 . 
La figure 4 correspond à des fils de $1 / 10 \mathrm{~mm}$ et la figure 5 à des fils de $5 / 10 \mathrm{~mm}$. On constate que dans le premier cas l'effet de puits est effectivement négligeable mais qu'il faut le prendre en considération dans le deuxième cas, si la durée de l'expérience dépasse une certaine limite. Il en résulte que - si pour des raisons de tenue mécanique on décide d'adopter des fils de $5 / 10 \mathrm{~mm}$ - il ne faudra utiliser pour les dépouillements qu'une partie des enregistrements. Ces résultats nous ont conduits à ajouter une protection supplémentaire contre les fuites thermiques en utilisant des gaines thermorétractables.

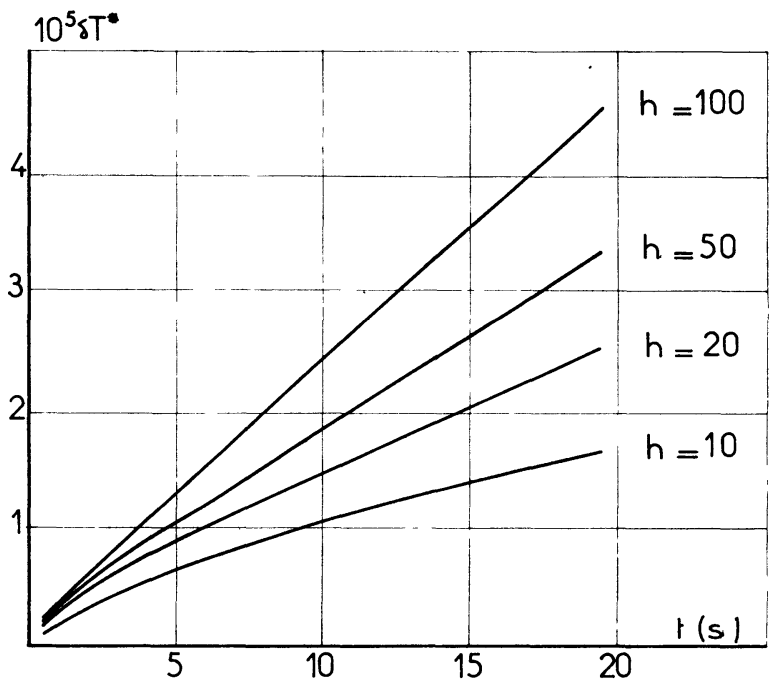

Fig. 4. - Effet de puits caractérisé par $\left[\frac{T(\mathrm{C} \mid . t)-T(\mathrm{C} \mid .0)}{T(\mathrm{C} \mid .0)}\right]_{\alpha=0}=T_{*}$

pour des fils alumel de $1 / 10 \mathrm{~mm}$ dans le cas où $\gamma(C) . e(C)=5,37$.

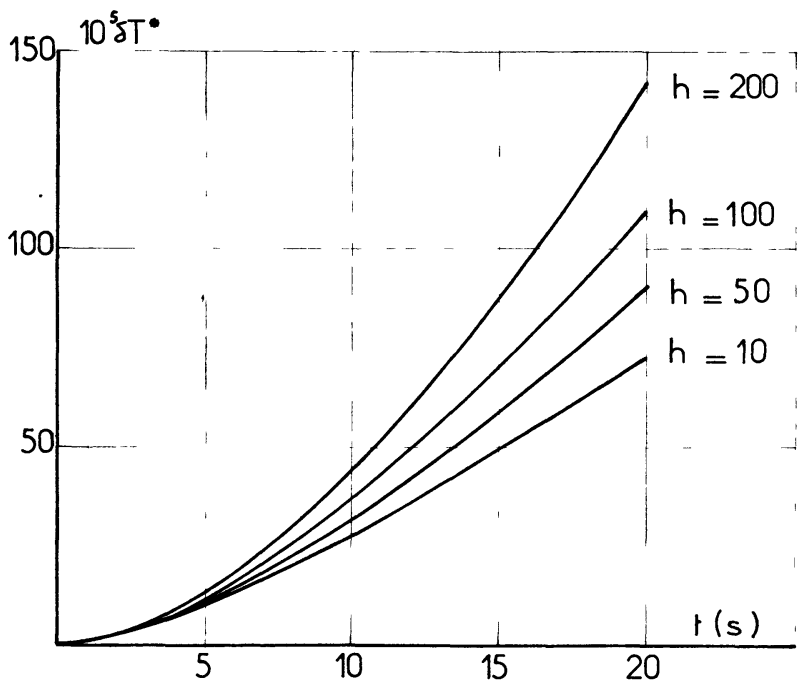

Fig. 5. - Effets de puits pour des fils de $5 / 10 \mathrm{~mm}$ dans le cas où $\gamma(\mathrm{C}) . e(\mathrm{C})=5,37$.

3. Etude sur la trempe de pièces métalliques. - Le deuxième problème concret que nous avons retenu concerne un dispositif mis au point à l'Ecole Nationale Supérieure de Métallurgie de l'Université de Nancy, en vue de définir les conditions dans lesquelles un milieu de trempe permet d'assurer de la façon la plus convenable le refroidissement d'une pièce métallique. Ce dispositif est schématisé sur la figure 6 extraite de la thèse citée en référence [5] dans laquelle l'auteur a présenté les résultats qu'il avait obtenus au sujet de la trempe d'éprouvettes en aluminium et en nickel.

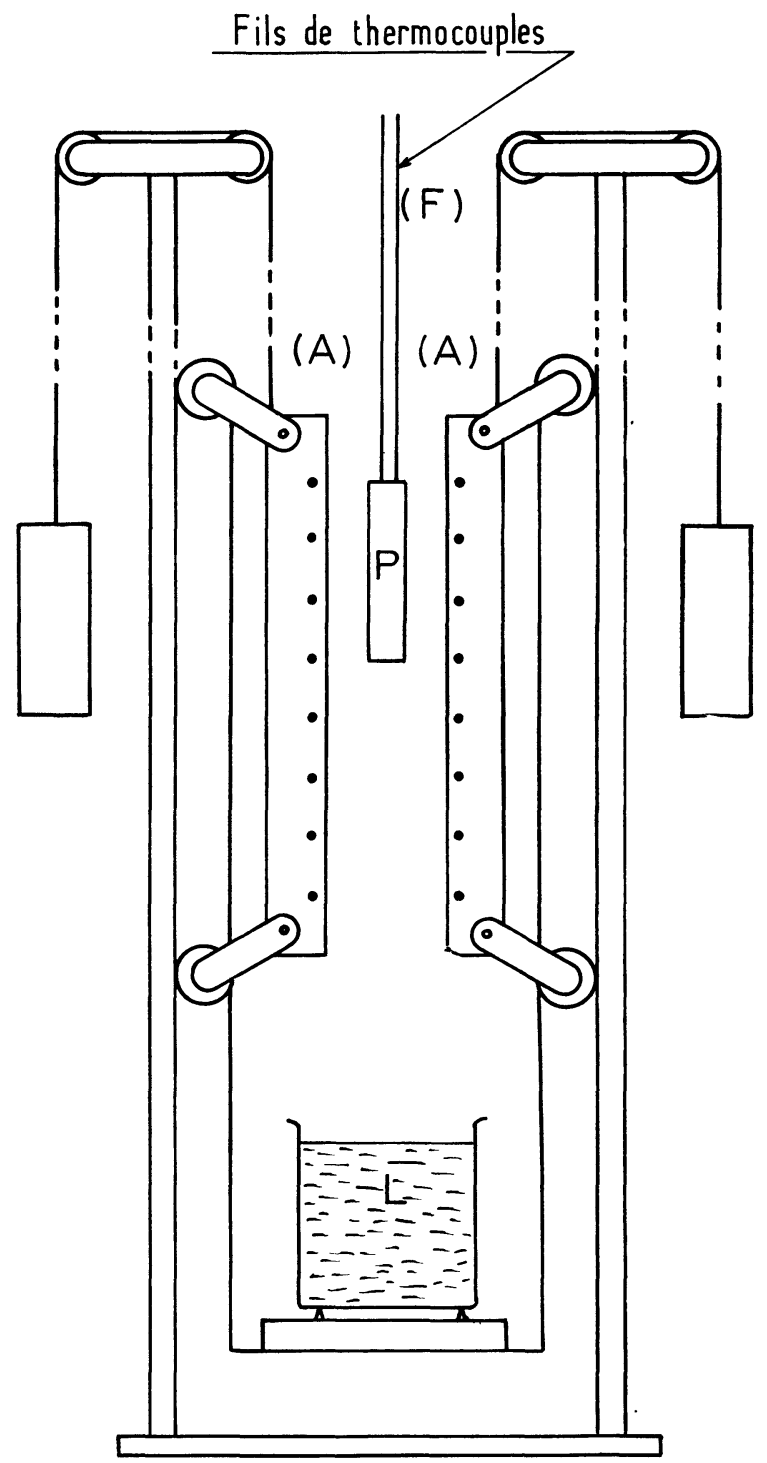

Fig. 6. - Dispositif d'études sur la trempe.

La pièce « $P$ » est placée au centre d'un four, où elle atteint un état d'équilibre thermique au niveau $T(\mathrm{P} \mid .0)$. Un mécanisme approprié permet alors de remplacer très rapidement le four par un bac de trempe contenant le liquide « $\mathrm{L}$ ». Au moment de l'immersion, deux liaisons thermiques s'établissent :

- l'une entre la pièce " $P$ » et le liquide " $L$ ",

- l'autre entre les fils de mesure « $F$ » et l'atmosphère ambiante " $A$ ». Nous admettrons comme précédemment qu'il s'agit d'échanges Newtoniens respectivement caractérisés par les paramètres

$$
\begin{array}{ll}
h(\mathrm{P} \leftrightarrow \mathrm{L}) \text { et } T(\mathrm{~L}) & \text { d'une part } \\
h(\mathrm{~F} \leftrightarrow \mathrm{A}) \text { et } T(\mathrm{~A}) & \text { d'autre part. }
\end{array}
$$


Nous désignerons par $s(\mathrm{P})$ la surface totale de la pièce « $\mathrm{P} »$, par $v(\mathrm{P})$ son volume et par $\gamma(\mathrm{P})$ la capacité calorifique volumique de sa substance et nous poserons :

$$
\begin{aligned}
\frac{h(\mathrm{P} \rightarrow \mathrm{L})}{\gamma(\mathrm{P})} \cdot \delta(\mathrm{P}) & =\beta \quad \pi \frac{[d(\mathrm{~F})]^{2} b(\mathrm{~F})}{\gamma(\mathrm{P}) \cdot V(\mathrm{P})}=\eta \\
m^{2} & =\frac{4 h(\mathrm{~F} \rightarrow \mathrm{A})}{\gamma(\mathrm{F}) d(\mathrm{~F})} .
\end{aligned}
$$

Au temps « $t$ » compté à partir de l'immersion, la température moyenne de la pièce dont on néglige comme précédemment la résistance interne est $T(\mathrm{P} \mid t)$. $\mathrm{Si}$ les fils de thermocouples n'existaient pas, cette fonction serait définie par la loi exponentielle

$$
\begin{aligned}
& \frac{\left.T_{\mathrm{r}}(\mathrm{P}) \mid . t\right)-T(\mathrm{P} \mid .0)}{T(\mathrm{P} \mid .0)}= \\
& \quad=\frac{T(\mathrm{~L})-T(\mathrm{P} \mid .0)}{T(\mathrm{P} \mid .0)}[1-\exp (-\beta t)] .
\end{aligned}
$$

Si l'on traduit - dans l'espace « p » de Laplace - le bilan thermique de l'ensemble des quatre éléments « $\mathrm{P} »$, , $\mathrm{L} »$, , $\mathrm{F} »$, , $\mathrm{A}$ », on obtient les expressions

$$
\begin{aligned}
& \quad \mathcal{L} \varphi(\mathrm{P} \rightarrow \mathrm{F})= \\
& =b(\mathrm{~F})\left[\mathcal{L} T(\mathrm{P} \mid . t)-\frac{T(\mathrm{P} \mid \cdot 0)+m^{2} T(\mathrm{~A})}{p+m^{2}}\right] \cdot \sqrt{p+m^{2}}
\end{aligned}
$$

$$
\begin{aligned}
& \mathcal{L}\left[\frac{T(\mathrm{P} \mid . t)-T(\mathrm{P} \mid .0)}{T(\mathrm{P} \mid .0)}\right]= \\
= & \beta \frac{T(\mathrm{~L})-T(\mathrm{P} \mid .0)}{T(\mathrm{P} \mid .0)} \frac{1}{p\left(p+\beta+\eta \sqrt{p+m^{2}}\right)}- \\
& -\frac{T(\mathrm{P} \mid .0)-T(\mathrm{~A})}{T(\mathrm{P} \mid .0)} \frac{\eta m^{2}}{p \sqrt{p+m^{2}}\left[p+\beta+\eta \sqrt{p+m^{2}}\right]}
\end{aligned}
$$

qui font intervenir les trois paramètres $\beta, \eta$ et $m$. Pour en déduire le refroidissement relatif

$$
\frac{T(\mathrm{P} \mid .0)-T(\mathrm{P} \mid . t)}{T(\mathrm{P} \mid .0)}=\delta T_{*}
$$

de la pièce au cours de la trempe, on effectue dans l'espace $\left\langle p »\right.$ la translation $p+m^{2}=p^{\prime}$.

L'expression $p+\beta+\eta \sqrt{p+m^{2}}$ a pour homologue

$$
p^{\prime}+\eta q^{\prime}+\beta-m^{2}=q^{\prime 2}+\eta q^{\prime}+\beta-m^{2}=f\left(q^{\prime}\right)
$$

et, étant donné l'ordre de grandeur relatif des trois paramètres $\eta$, $\beta$ et $m^{2}$ l'équation $f\left(q^{\prime}\right)=0$ admet deux racines réelles $q_{1}^{\prime}$ et $q_{2}^{\prime}$.

Il est alors facile d'effectuer un développement de l'expression (4) en éléments simples correspondant aux pôles $0, q_{1}^{\prime}$ et $q_{2}^{\prime}$ et d'inverser les différents termes de la formule. Cette inversion fait intervenir les fonctions

$$
\left[\exp \left(-m^{2} t\right) \Theta(m \sqrt{t})\right], \exp \left(q^{2} t\right) \text { et } \Theta(q \sqrt{t})
$$

dont on possède des tables numériques très complètes. La formule qui donne explicitement l'expression de $\delta T_{*}$ est longue. Aussi ne croyons nous pas utile de la reproduire.

Son traitement numérique permet d'établir des graphiques fournissant pour des valeurs données des paramètres $\eta, \beta$ et pour différentes valeurs du coefficient d'échange $h(\mathrm{~F} \leftrightarrow \mathrm{A})$ la variation en fonction du temps de la fonction

$$
\delta T_{*}=\frac{T_{\mathrm{r}}(\mathrm{P} \mid \cdot t)-T_{\mathrm{m}}(\mathrm{P} \mid . t)}{T(\mathrm{~L})-T(\mathrm{P} \mid .0)}
$$

où $T_{\mathrm{r}}(\mathrm{P} / \mathrm{T})$ représente la température qu'aurait la pièce dans une opération réelle detre mpe (formule (2)) et $T_{\mathrm{m}}(\mathrm{P} / \mathrm{T})$ la température déduite des enregistrements obtenus dans les expériences effectuées sur une pièce équipée à l'aide de capteurs à fils (formule 4 inversée).

A titre d'exemple, nous avons reproduit sur la figure 7 l'allure générale d'un de ces réseaux. Pour un thermocouple chromel alumel de $5 / 10$ isolé sous soie paraffinée, on peut admettre que dans les conditions de l'expérience, le coefficient $h$ est de l'ordre de 100. Comme la durée de l'expérience est de l'ordre de $20 \mathrm{~s}$, on voit d'après le graphique, qu'il n'y a pas lieu d'effectuer la moindre correction.

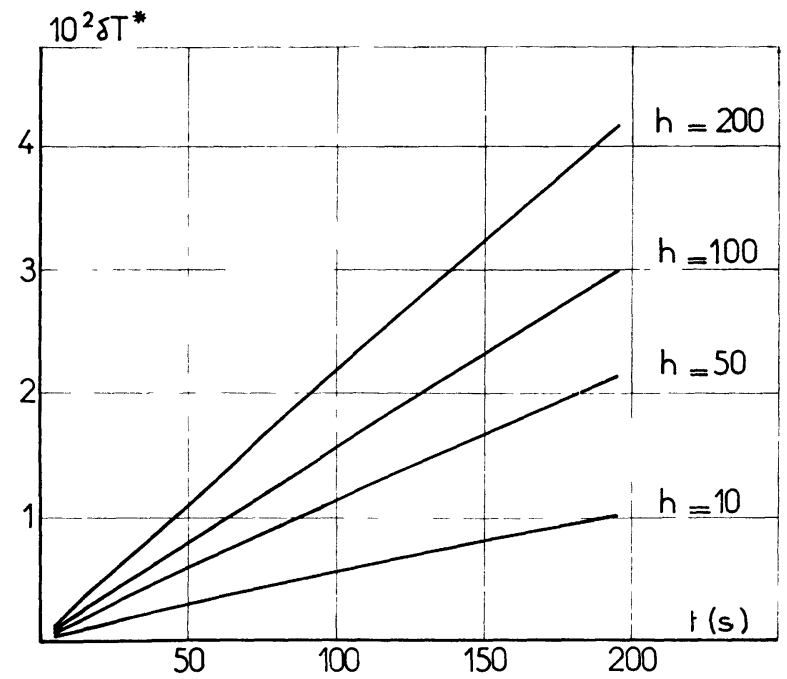

Fig. 7. - Effets de puits dans le dispositif de trempe (couple chromel-alumel de diamètre $d=5 / 10 \mathrm{~mm}$, capacité calorifique 5,37 . Surface de la pièce $S=9,5 \times 10^{-4}$ coefficient d'échange $h(\mathrm{P} \leftrightarrow \mathrm{L})=100$.

En conclusion, on pouvait sans inconvénient utiliser des fils de gros diamètre, à condition toutefois de prendre soin à leur isolation.

4. Etude sur l'introduction des gaz dans un réacteur. - Le dernier exemple que nous avons retenu se rapporte à la cinétique chimique. Il nous a été sug- 
géré par l'examen d'un dispositif utilisé au laboratoire d'Echanges Thermiques de l'Université de Paris VI pour étudier les phénomènes qui accompagnent l'introduction dans un réacteur de gaz stockés sous pression [6]. La partie du dispositif qui nous intéresse ici est schématisée sur la figure 8. Dans certains cas le réacteur a une forme cylindrique et dans d'autres une forme sphérique. La jonction thermoélectrique y est positionnée grâce à une gaine en alumine frittée. La tête de mesure peut être assimilée à une sphère de diamètre $D(\mathrm{C})$. Les données numériques extraites des publications [7] et [8] nous conduisent à évaluer à $5 \times 10^{3}$ le coefficient d'échange $h(\mathrm{G} \rightarrow \mathrm{C})$ entre le gaz introduit dans le réacteur et cette tête de mesure.
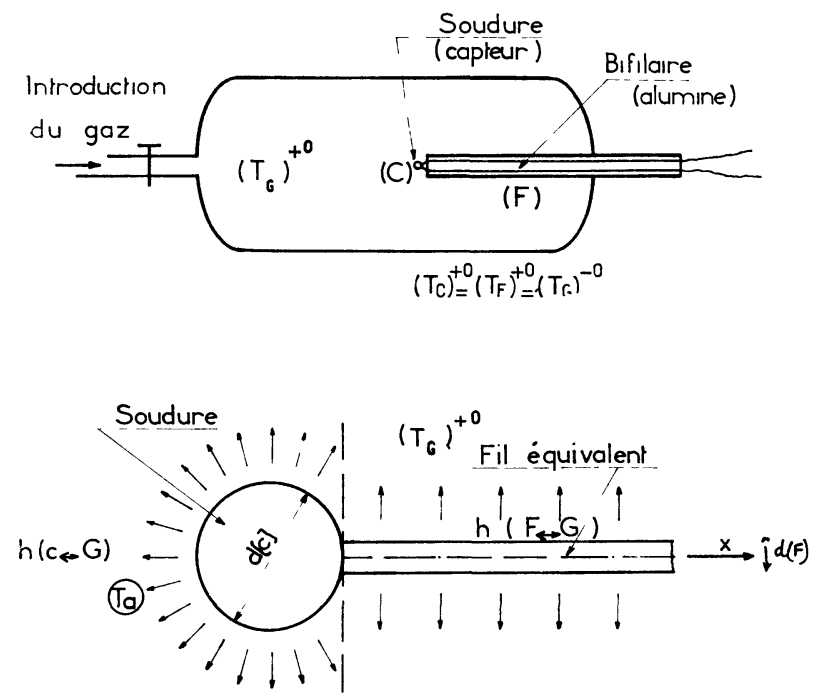

FIG. 8. - Dispositif d'étude de cinétique chimique.

Pour représenter les phénomènes thermiques qui accompagnent l'introduction du gaz dans le réacteur nous avons envisagé divers types de programmation en températures $T(\mathrm{G} \mid \cdot t)$ et comparé les résultats ainsi obtenus avec les résultats observés.

Le modèle thermocinétique le plus simple et le plus suggestif repose sur l'assimilation de cette programmation à un échelon d'amplitude $\delta T(\mathrm{G})$. Les calculs correspondants sont analogues, dans leur principe, à ceux que nous avons indiqués à propos des expériences sur la trempe des métaux, les conditions initiales étant définies par :

$$
\begin{aligned}
& T(\mathrm{C} \mid \cdot+0)=T(\mathrm{~F} \mid \cdot+0)=T(\mathrm{G} \mid \cdot-0) \\
& T(\mathrm{G} \mid \cdot+0)=T(\mathrm{G} \mid \cdot-0)+\delta T(\mathrm{G}) .
\end{aligned}
$$

Les thermogrammes dépendent comme nous l'avons vu de trois paramètres $\beta, \eta$ et $m$, mais dans le cas actuel, les paramètres $\beta$ et $\eta$ ont le même ordre de grandeur si bien que l'équation résolvante :

$$
q^{\prime 2}+\eta q^{\prime}+\beta-m^{2}=0
$$

admet des racines complexes. Pour obtenir un schéma explicite il faut alors utiliser un développement de la fonction $\Theta(z)$ en série d'arguments complexes, ce qui entraîne certaines difficultés d'une part à cause des erreurs d'arrondis propres à la machine et, d'autre part, parce qu'on ne peut pas utiliser en raccordement le développement asymptotique $\operatorname{de} \exp \left(z^{2}\right)[1-\Theta(z)]$ car la série résultante est oscillante.

Fort heureusement, ces difficultés correspondent à la période d'extinction de l'évolution thermique dont l'étude présente de l'intérêt sur le plan théorique mais qui n'était pas prise en considération dans l'étude du problème concret, que nous analysons.

Pour optimiser le dispositif métrologique nous avons donc considéré différents coefficients d'échange $h(\mathrm{G} \rightarrow \mathrm{C})$ entre le gaz « $\mathrm{G}$ » introduit dans le réacteur et la jonction thermoélectrique " $\mathrm{C}$ » et pour chacun d'eux nous avons construit un réseau de courbes représentant la correspondance

$$
t \leftrightarrow \frac{T_{\mathrm{r}}-T_{\mathrm{m}}}{\delta T(\mathrm{G})}=\Theta^{*}
$$

pour différentes valeurs du coefficient d'échange $h(\mathrm{G} \rightarrow \mathrm{F})$ entre le gaz « $\mathrm{G}$ » et le dispositif de mesure « $F$ ». La figure 9 reproduit le réseau qui correspond à $h(\mathrm{G} \rightarrow \mathrm{C})=5000$. Les limites $h=0$ et $h=5000$ des cotes correspondent respectivement à un isolement parfait des fils et à des fils nus.

On voit que pour les faibles valeurs de $h$ la grandeur

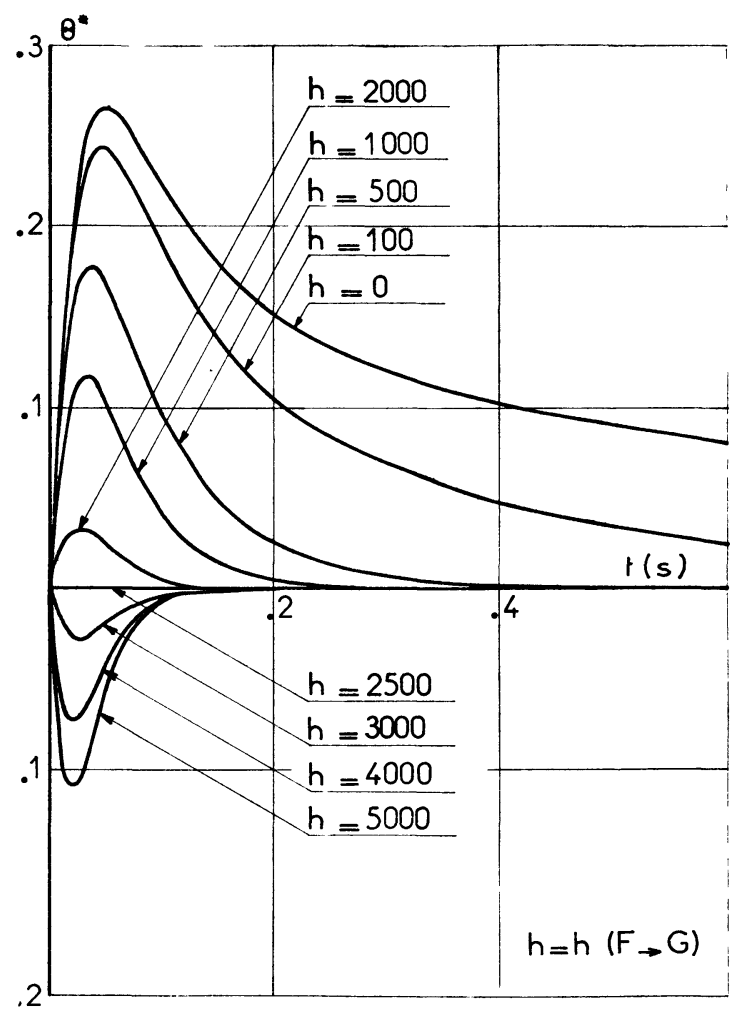

FIG. 9. - Adaptation thermique du capteur à une programmation en échelon :

$$
\left\{\begin{array}{l}
d(\mathrm{C})=0,15 \mathrm{~mm} \quad \gamma(\mathrm{C})=7,4 \times 10^{-6} \\
d(\mathrm{~F})=\sqrt{2} .50 \mu \mathrm{m} \\
h(\mathrm{C} \leftrightarrow \mathrm{G})=5000 .
\end{array}\right.
$$


adimensionnelle $\Theta$ présente, pour une certaine valeur de $t$, un maximum dont l'amplitude décroît lorsque $h$ augmente. Pour les grandes valeurs de $h$ elle présente au contraire un minimum dont l'amplitude diminue lorsque $h$ diminue. La jonction entre les deux réseaux de courbes s'effectue pour une certaine valeur $h_{\mathrm{c}}$ qui, dans l'exemple précis considéré, était égale à 2500 .

Pour interpréter ces résultats il est logique, dans le cas actuel, de substituer aux paramètres considérés précédemment les deux constantes de temps $\tau(\mathrm{C})$ et $\tau(F)$ définies par :

$$
\tau(\mathrm{C})=\frac{\gamma(\mathrm{C}) \cdot D(\mathrm{C})}{6 h[\mathrm{C} \rightarrow \mathrm{G}]} \quad \text { et } \quad \tau(\mathrm{F})=\frac{\gamma(\mathrm{F}) \cdot D(\mathrm{~F})}{4 h(\mathrm{~F} \rightarrow \mathrm{G})}
$$

[sphère et cylindre].

Dans les expériences effectuées, la constante de temps $\tau(C)$ était de l'ordre de $0,025 \mathrm{~s}$ et la constante de temps $\tau(\mathrm{F})$ variait entre $0,01 \mathrm{~s}$ (fil $\mathrm{nu}$ ) et $5,25 \mathrm{~s}$ (fil isolé de coefficient $h=10$ ). La constante de temps des fils est supérieure à celle du capteur pour les échanges dont le coefficient est inférieur à 2500 . Elle lui est supérieure dans les autres cas. Ces considérations permettent de comprendre l'allure générale du réseau $t \leftrightarrow \Theta_{*}$.

Si le dispositif de mesure est constitué par un thermocouple, les deux paramètres $\gamma(\mathrm{F})$ et $\gamma(\mathrm{C})$ sont égaux et le rapport des constantes de temps considérées s'exprime sous la forme

$$
\frac{\tau(\mathrm{F})}{\tau(\mathrm{C})}=\frac{3}{2} \frac{D(\mathrm{~F})}{D(\mathrm{C})} \cdot \frac{h(\mathrm{C} \rightarrow \mathrm{G})}{h(\mathrm{~F} \rightarrow \mathrm{G})}
$$

Il est donc égal au rapport des coefficients d'échange lorsque les diamètres respectifs de la tête de mesure et du fil de liaison sont entre eux dans le rapport $3 / 2$.

Dans ce cas la perturbation apportée par les fils est minima lorsque les deux coefficients d'échange sont égaux c'est-à-dire lorsque les fils ne sont pas isolés dans une gaine.

On voit - en tenant compte des autres dispositions technologiques - que l'on a intérêt, au point de vue thermométrique, de choisir des fils de liaison aussi fins et aussi peu isolés que possible.

Dans l'appareil construit au Laboratoire on a adopté des fils de $50 \mu$.
5. Conclusion. - Pour tirer des conclusions pratiques de ces trois études particulières, nous allons en résumer les résultats : dans le cas du contact solidesolide, illustré par l'appareil d'études de l'effusivité, la perturbation apportée par l'existence de fils de liaison entre le capteur et la chaîne de mesure est d'autant plus réduite que la durée de l'expérience est plus faible et les fils mieux isolés. Pour optimiser le dispositif expérimental en tenant compte des autres facteurs, on peut adopter des diamètres de fil relativement importants. Toutefois, on ne peut pas dépasser sans risque un certain seuil (de l'ordre de $2 / 10 \mathrm{~mm}$ ) qu'après avoir effectué un calcul numérique complet basé sur la considération du bilan global.

On remarque, en outre, que la perturbation apportée par le dispositif de mesure est très notablement inférieure à celle que l'on obtiendrait en régime établi.

Dans le cas du contact solide-liquide, illustré par l'étude du phénomène de trempe, nous avons montré que l'on pouvait utiliser sans précaution des fils de gros diamètres pour des expériences relativement longues à condition d'isoler convenablement ces fils.

Enfin, dans le cas du contact solide-gaz, illustré par l'étude du réacteur, l'optimisation du dispositif conduisait à adopter des fils aussi fins et aussi peu isolés que possible.

Ces résultats, très différents les uns des autres, montrent que dans la thermométrie par contact, il n'existe pas de recette universelle. Chaque problème nécessite une étude particulière. Pour l'effectuer, il est nécessaire d'élaborer un modèle thermocinétique convenablement adapté. Parfois, on peut en majorant certains termes, obtenir des renseignements suffisants en utilisant simplement des tables numériques usuelles. Parfois, au contraire, il est nécessaire d'effectuer une analyse plus fine, à la fois au point de vue analytique et au point de vue calcul. Pour chaque type de détecteurs on peut alors - en utilisant des calculs par ordinateur - construire des abaques qui permettent de répondre aux questions posées.

Remarquons, pour terminer, que les formules obtenues s'appliquent non seulement aux détecteurs constitués par des couples thermoélectriques mais aussi aux autres types de détecteurs : thermistances et sondes à résistance. Les caractéristiques du fil équivalent sont, même dans ces cas, beaucoup mieux définies qu'elle ne l'étaient pour les thermocouples.

\section{Bibliographie}

[1] Poncin, H., Les apports de la thermocinétique à la détermination des températures de surface. Cahiers de la thermique (I. F. C. E. 1972-4-123)

[2] Watson, G. G., Techniques for measuring surface temperature Inst. Pract. (1966-6-217).

[3] Bransier, J., Contribution à l'étude de l'effusivité thermique (Doctorat de spécialité, Paris 1972).

[4] BRANSIER, J., International Journal of Heat and mass transfer 17 (1974) 498.
[5] Chevrier, J. C., Thèse de Doctorat és Sciences Physiques, Université de Nancy I, 1973.

[6] Ben Aim-Bicquart, J., Doctorat de spécialité, Paris 1972 et Bulletin de la Société Chimique de France 1972, $\mathrm{n}^{\circ} 11$, p. 4114.

[7] Arques, P., Bibliothèque du Laboratoire de Mécanique Physique de Saint-Cyr. Rapport interne 1971.

[8] Laufer, J. et Mc Clellan, J. Fluid. Méc. 1 (1956) 276. 